\title{
Моделирование диффузионно-дрейфового транспорта носителей заряда в полупроводниковых слоях с фрактальной структурой в переменном электрическом поле
}

\author{
(C) С.Ш. Рехвиашвили, А.А. Алиханов \\ Институт прикладной математики и автоматизации КБНЦ Российской академии наук, \\ 360000 Нальчик, Россия \\ E-mail: rsergo@mail.ru
}

(Получена 22 ноября 2016 г. Принята к печати 23 ноября 2016 г.)

На основе дифференциального уравнения в частных производных дробного порядка проведено моделирование диффузионно-дрейфового транспорта носителей заряда в полупроводниковом слое с фрактальной структурой под действием продольного переменного электрического поля. В рамках модели показано, что в слоях с фрактальной структурой имеют место уширение и асимметрия пространственно-временны́х распределений носителей заряда. При определенных условиях наблюдается эффект удвоения частоты осцилляций заряда во внешнем переменном электрическом поле.

DOI: 10.21883/FTP.2017.06.44558.8433

\section{1. Введение}

Искусственные и природные материалы с фрактальной структурой являются нерегулярными фракталами, которые сохраняют свойство самоподобия в ограниченных пространственных масштабах — от отдельных атомов или кластеров до агрегата в целом (так называемые „предфракталы“). Слои конденсированных веществ с фрактальной структурой и различными типами межатомных связей могут быть получены с помощью таких технологий, как газофазное и жидкофазное эпитаксиальное наращивание, электролиз, магнетронное распыление, молекулярно-лучевое наслаивание, золь-гель технология и др. Такие слои являются перспективными для применения в микро- или наноэлектронике для создания чувствительных оптических и газовых сенсоров, термоизолирующих покрытий, а также приборов на квантово-размерных эффектах (например, пористый кремний). К материалам с фрактальной структурой можно отнести разнообразные дендритные кристаллы, образование которых обусловлено резким переохлаждением и большой скоростью кристаллизации. В настоящее время уже стало ясно, что адекватное описание физикохимических свойств дендритных кристаллов невозможно без применения понятия фрактала. С развитием нанотехнологий появилась также возможность синтезировать и визуализировать отдельные молекулы с фрактальной структурой.

В связи с вышесказанным большой интерес представляет теоретическое исследование особенностей транспорта носителей заряда (Н3) во фрактальных полупроводниковых слоях. Одним из результативных теоретических подходов здесь оказался подход, основанный на применении дробного интегро-дифференцирования [1-5]. В работе [6] предложена новая теоретическая модель диффузионно-дрейфового транспорта Н3, основанная на применении оператора дробного интегро- дифференцирования Капуто порядка $\alpha \in(0,1]$. Было найдено в аналитическом виде решение задачи Коши для дифференциального уравнения модели при условии, что внешнее электрическое поле является постоянным. На практике, однако, эксперименты по исследованию проводящих материалов часто проводят с использованием переменных электрических полей. При этом возникает ряд новых особенностей, в частности в переменном электрическом поле дрейфовая скорость может не совпадать по фазе с напряженностью поля и подвижность НЗ зависит от частоты поля.

В данной статье порядок дробного интегро-дифференцирования в уравнении диффузии связывается с показателем Херста и фрактальной размерностью траектории случайного блуждания; с использованием модели, предложенной в работе [6], проводится численное моделирование диффузионно-дрейфового транспорта Н3 во фрактальном полупроводниковом слое под действием продольного переменного электрического поля.

\section{2. О применении дробного интегро-дифференцирования}

Математический аппарат дробного интегро-дифференцирования играет конструктивную роль во фрактальном анализе [1-3]. Для физической интерпретации дробного интегро-дифференцирования в рассматриваемой задаче проанализируем модель случайных блужданий частицы в отсутствие внешних полей. В работе [6] и в данной работе используется дробный интеграл РиманаЛиувилля [7,8]:

$$
D_{0 t}^{\alpha-1} x(t)=\frac{1}{\Gamma(1-\alpha)} \int_{0}^{t} \frac{x\left(t^{\prime}\right) d t^{\prime}}{\left(t-t^{\prime}\right)^{\alpha}}, \quad 0<\alpha \leq 1,
$$


а также дробная производная Капуто:

$$
\partial_{0 t}^{\alpha} x(t)=D_{0 t}^{\alpha-1} \frac{d x(t)}{d t},
$$

где $\Gamma(z)-\gamma$-функция Эйлера, $\alpha-$ порядок дробного интегро-дифференцирования. Заметим, что если $x(0)=0$, то справедливо тождество: $D_{0 t}^{\alpha} x(t) \equiv$ $\equiv \partial_{0 t}^{\alpha} x(t)[7,8]$.

Рассмотрим процесс диффузии частиц в среде, которая является стохастическим фракталом. Траектория движения отдельной частицы представляет собой всюду недифференцируемую фрактальную (самоафинную) кривую $[9,10]$. Для зависящих от времени фрактальных функций, в том числе и для траектории броуновского движения, не представляется возможным вводить скорость как первую производную по времени. В связи с этим в работах $[6,11,12]$ в различных задачах вместо обычной локальной скорости предложено рассматривать эффективную скорость движения частицы в среде с использованием понятия дробного интегродифференцирования. Для описания случайного блуждания частиц здесь будем рассматривать средние значения модуля смещения частицы по времени и ансамблю. Согласно $[6,11,12]$, для эффективной скорости имеем

$$
\begin{aligned}
\langle v\rangle=\frac{1}{\tau} D_{0 t}^{H-1} \frac{d\langle x(t)\rangle_{t}}{d t} & =\frac{1}{\tau} \partial_{0 t}^{H}\langle|x(t)|\rangle_{t}, \quad 0<H \leq 1 / 2, \\
\langle|x(t)|\rangle_{t} & =\frac{1}{t} \int_{0}^{t}|x(t)| d t,
\end{aligned}
$$

где $x(t)$ - координата частицы, $t-$ безразмерное (отнесенное к $\tau$ ) время, $\tau-$ некоторая постоянная с размерностью времени, смысл которой определяется далее, $H$ - параметр, отвечающий за эффект памяти. Время $\tau$ вводится в формулу (1) для приведения дробных операторов к безразмерному виду. В формуле (1) оператор дробного интегрирования $D_{0 t}^{H-1}$ учитывает наличие в системе памяти $[1,2]$.

Задача заключается в том, чтобы придать физический смысл скорости $\langle v\rangle$ и параметру $H$. Если предположить, что в отсутствие полей $\langle v\rangle=$ const, то из (1) получим

$$
\langle|x(t)|\rangle_{t}=\tau \int_{0}^{t} D_{0 t}^{1-H}\langle v\rangle d t=\tau D_{0 t}^{-H}\langle v\rangle=\frac{\tau\langle v\rangle}{\Gamma(1+H)} t^{H} .
$$

Запишем теперь в безразмерных переменных дробное уравнение диффузии с начальным условием в виде $\delta$-функции Дирака:

$$
\begin{gathered}
\partial_{0 t}^{2 H} f(x, t)=\frac{\partial^{2} f(x, t)}{\partial x^{2}}, \quad 0<H \leq 1 / 2, \\
f(x, 0)=\delta(x), \\
f(+\infty, t)=f(-\infty, t)=0,
\end{gathered}
$$

где $f(x, t)$ - плотность распределения, $\delta(x)-\delta$-функция Дирака. Время $t$ и координата $x$ в уравнении (3) отнесены соответственно к $\tau$ и $\sqrt{D \tau}$, где $D-$ коэффициент диффузии. Различные начально-краевые задачи для уравнений в частных производных дробного порядка в настоящее время достаточно хорошо исследованы (см. работы $[8,13]$ и ссылки в них). Используя, в частности, результаты работ $[8,13]$, можно выписать решение задачи (3) в виде

$$
\begin{gathered}
f(x, t)=0.5 t^{-H} \phi\left(-H, 1-H,-|x| t^{-H}\right), \\
\phi(\alpha, \beta, z)=\sum_{n=0}^{\infty} \frac{z^{n}}{n ! \Gamma(\alpha n+\beta)},
\end{gathered}
$$

где $\phi(\alpha, \beta, z)$ - функция Райта. Учитывая свойства функции Райта [8] и переходя к размерной координате $x$, для среднего модуля смещения с учетом (4) находим

$$
\langle|x(t)|\rangle_{a}=\int_{-\infty}^{\infty}|x| f(x, t) d x=\frac{\sqrt{D \tau}}{\Gamma(1+H)} t^{H} .
$$

Формулы (2) и (5) идентичны, если выполняется равенство

$$
D=\tau\langle v\rangle^{2} .
$$

Если для средней скорости и подвижности частиц принять хорошо известные из молекулярной физики формулы $\langle v\rangle=\sqrt{k T / m}$ и $\mu=\tau / m$, то из (6) точно следует соотношение Эйнштейна для коэффициента диффузии.

Из проведенного анализа следует, что параметр $H$ в (2) и (5) тождествен параметру Херста, который связан с фрактальной размерностью траектории броуновской частицы соотношением [9,10]: $d=2-H$. При $H=1 / 2$ имеет место классическая диффузия. При $H<1 / 2$ процесс диффузии происходит медленно (так называемая субдиффузия) и характеризуется антиперсистентностью [9]. Обратим также внимание на следующее важное обстоятельство. Интегрирование в (2) и (5) проводится соответственно по времени и координате, причем результаты этого интегрирования совпадают, если имеет место соотношение Эйнштейна (6). Физически это можно трактовать как выполнение эргодической гипотезы для рассмотренного случая. В реальности же выполнение этой гипотезы можно ожидать, если следить за блужданием частицы в течение очень большого промежутка времени $(t \rightarrow \infty)$.

В общем случае следует различать фрактальные блуждания в регулярных средах и блуждания на фракталах [14]. Применение уравнения (3) для моделирования блужданий на фракталах, по нашему мнению, возможно в силу следующих обстоятельств. Во-первых, представляется очевидным, что в однородной и макроскопически сплошной среде нет никаких причин для возникновения субдиффузионного режима; в такой среде всегда будет иметь место классическая диффузия при $H=1 / 2$. При наличии в среде фрактальных неоднородностей процесс диффузии в ней должен происходить медленнее, чем в классическом случае, что соответствует условию 
$H<1 / 2$. При этом, если система является эргодической, то параметр $H$ будет непосредственно характеризовать степень неоднородности среды. Для такой системы введение дробных производных по пространственным координатам, по-видимому, является избыточным. Эти рассуждения, по существу, согласуются с выводами относительно коэффициента диффузии в модели обобщенного броуновского движения [9]. Во-вторых, в модели дробной диффузии различие в эволюциях диффузионных пакетов в случаях блуждания в регулярной среде и блуждания на фрактале (см. [14]) при $t \rightarrow \infty$ и $x \rightarrow \infty$ может быть формально нивелировано путем подбора численных значений порядков дробного интегро-дифференцирования. В силу известной произвольности выбора значений этих порядков (физически для различных типов блужданий они, очевидно, не обязаны быть одинаковыми) в модели дробной диффузии, рассмотренной в работе [14], для фрактального блуждания в регулярной среде всегда можно поставить в соответствие определенное блуждание на фрактале.

\section{3. Моделирование диффузионно-дрейфового транспорта Н3}

В работе [6] выведено следующее уравнение, описывающее диффузионно-дрейфовый транспорт Н3 во фрактальном полупроводнике:

$$
\frac{1}{\tau} \partial_{0 t}^{\alpha} \rho+(\mu \mathbf{E}, \nabla \rho)-D \Delta \rho=q(G-R), \quad 0<\alpha \leq 1,
$$

где $\rho$ - объемная плотность заряда, $\mathbf{E}-$ вектор напряженности внешнего электрического поля, $G$ и $R-$ функции, которые характеризуют темпы генерации и рекомбинации Н3, $q$ - заряд электрона. Порядок дробной производной в (7) связан с показателем Херста так, как это показано в предыдущем разделе: $\alpha=2 H$. Предполагается, что внешнее электрическое поле не влияет на показатели $\alpha$ и $H$, которые характеризуют внешнюю фрактальную среду. В отсутствие процессов генерации и рекомбинации, а также при наличии внешнего переменного электрического, изменяющегося по гармоническому закону, из (7) получаем уравнение

$$
\begin{gathered}
\partial_{0 t}^{\alpha} \rho-\frac{\partial^{2} \rho}{\partial x^{2}}+a \cos (\omega t) \frac{\partial \rho}{\partial x}=0,-\infty<x<+\infty, t \geq 0 \\
a=\mu E \sqrt{\frac{\tau}{D}}=\text { const, }
\end{gathered}
$$

где $a$ и $\omega-$ безразмерные амплитуда и частота поля. В (8) использованы безразмерные переменные (время и координата отнесены к $\tau$ и $\sqrt{D \tau}$ ). Для уравнения (8) принимается начальное условие

$$
\rho(x, 0)=\rho_{0}(x), \quad-\infty<x<+\infty,
$$

где $\rho_{0}(x)$ определяет начальный профиль распределения НЗ.
Задача Коши (8),(9) решается численно с помощью метода, разработанного в [15]. Для оператора Капуто используется разностный аналог:

$$
\begin{gathered}
\Delta_{0 t_{j+\sigma}}^{\alpha} \rho_{i}=\frac{(\Delta t)^{-\alpha}}{\Gamma(2-\alpha)} \sum_{s=0}^{j} c_{j-s}^{(\alpha, \sigma)}\left(\rho_{i}^{s+1}-\rho_{i}^{s}\right), \\
\sigma=1-\alpha / 2, \quad a_{0}^{(\alpha, \sigma)}=\sigma^{1-\alpha}, \\
a_{l}^{(\alpha, \sigma)}=(l+\sigma)^{1-\alpha}-(l-1+\sigma)^{1-\alpha}, \\
b_{l}^{(\alpha, \sigma)}=\frac{1}{2-\alpha}\left[(l+\sigma)^{2-\alpha}-(l-1+\sigma)^{2-\alpha}\right] \\
-\frac{1}{2}\left[(l+\sigma)^{1-\alpha}+(l-1+\sigma)^{1-\alpha}\right], \quad l \geq 1, \\
c_{s}^{(\alpha, \sigma)}=\left\{\begin{array}{l}
c_{0}^{(\alpha, \sigma)}=a_{0}^{(\alpha, \sigma)}, \quad j=0, \\
a_{0}^{(\alpha, \sigma)}+b_{1}^{(\alpha, \sigma)}, \quad s=0, \\
a_{j}^{(\alpha, \sigma)}+b_{s+1}^{(\alpha, \sigma)}-b_{s}^{(\alpha, \sigma)}, \quad s=j, j \geq 1,
\end{array}\right.
\end{gathered}
$$

где $\Delta t$ - шаг по времени. Уравнение (8) представляется в дивергентной форме и с учетом формулы (10) в прямоугольнике $\bar{D}=\{(x, t):-l \leq x \leq l, 0 \leq t \leq T\}$ решается разностная схема:

$$
\begin{gathered}
\Delta_{0 t_{j+\sigma}}^{\alpha} \rho_{i}=\sigma \frac{\exp \left(-\mu_{j+\sigma}\right) \rho_{i+1}^{j+1}-\left(\exp \left(-\mu_{j+\sigma}\right)+\exp \left(\mu_{j+\sigma}\right)\right) \rho_{i}^{j+1}+}{+\exp \left(\mu_{j+\sigma}\right) \rho_{i-1}^{j+1}} \\
+(1-\sigma) \frac{\exp \left(-\mu_{j+\sigma}\right) \rho_{i+1}^{j}-\left(\exp \left(-\mu_{j+\sigma}\right)+\exp \left(\mu_{j+\sigma}\right)\right) \rho_{i}^{j}+}{+\exp \left(\mu_{j+\sigma}\right) \rho_{i-1}^{j}}
\end{gathered}
$$

где $\Delta x$ - шаг по координате. Величина параметра $l$ подбирается так, чтобы обеспечить требуемую точность расчетов, соответствующую порядку точности разностного аналога оператора Капуто (10). В нашей модели „точка суперсходимости“ $\sigma$ разностной схемы (11) оказывается связанной с показателем Херста: $\sigma=1-H$.

При моделировании предполагалось, что начальный профиль распределения неравновесных НЗ имеет гауссову форму: $\rho_{0}(x)=A \exp \left(-B x^{2}\right)$, где $A$ и $B-$ положительные константы. Такой профиль может быть получен, например, импульсным воздействием лазерного пучка c $\mathrm{TEM}_{00}$ модой и гауссовым распределением интенсивности по сечению. В расчетах было принято $A=1 / \sqrt{\pi}$, $B=1$. 
Указанное значение множителя $A$ выбрано с целью проверки в расчетах с заданной точностью условия сохранения заряда:

$$
\int_{-l}^{l} \rho(x, t) d x=1 .
$$

Согласно работам $[1,16]$, показатель $\alpha$ соответствует доле проводящих каналов фрактальной системы. Следовательно, с уменьшением $\alpha$ проводимость фрактальной системы в целом должна уменьшаться, что соответствует данной в предыдущем разделе интерпретации дробного интегро-дифференцирования. В нашем случае низким значениям показателя $\alpha(\alpha<1$ или $H<1 / 2)$ отвечает диффузионно-дрейфовый транспорт в полупроводниковом слое, который может состоять из перколяционных кластеров, дендритных фрактальных деревьев или пористых материалов.

На рис. 1 показаны примеры расчета профилей распределения плотности НЗ при различных $t$. В переменном электрическом поле возникают пространственные осцилляции плотности распределения Н3, причем характер этих осцилляций критически зависит от параметра $\alpha$. В отсутствие рекомбинации под действием переменного электрического поля со временем происходит медленное рассасывание Н3 в объеме образца. При малых значениях $a$ и $\alpha$ наблюдаются асимметрия и „тяжелые хвосты“ распределений НЗ, что качественно согласуется с результатами работы [5]. Моделирование также показывает, что с увеличением амплитуды поля дрейфовая составляющая тока возрастает и эффекты, обусловленные наличием памяти, перестают быть заметными.

Численные расчеты при малых значениях параметров $a$ и $\alpha$ позволили выявить новый кинетический эффект, обусловленный специфической инерционностью Н3 во фрактальном слое и существованием степенной памяти. В субдиффузионном режиме при $\alpha<0.5$ рассасывание Н3 заряда существенно замедляется, что

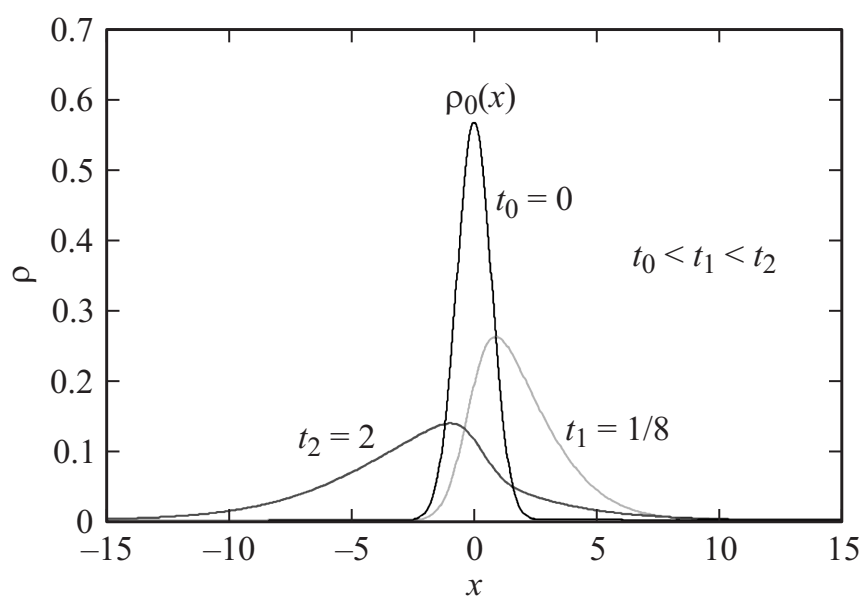

Рис. 1. Пространственные распределения плотности НЗ при $\alpha=0.5$ и $\omega=a=5$.
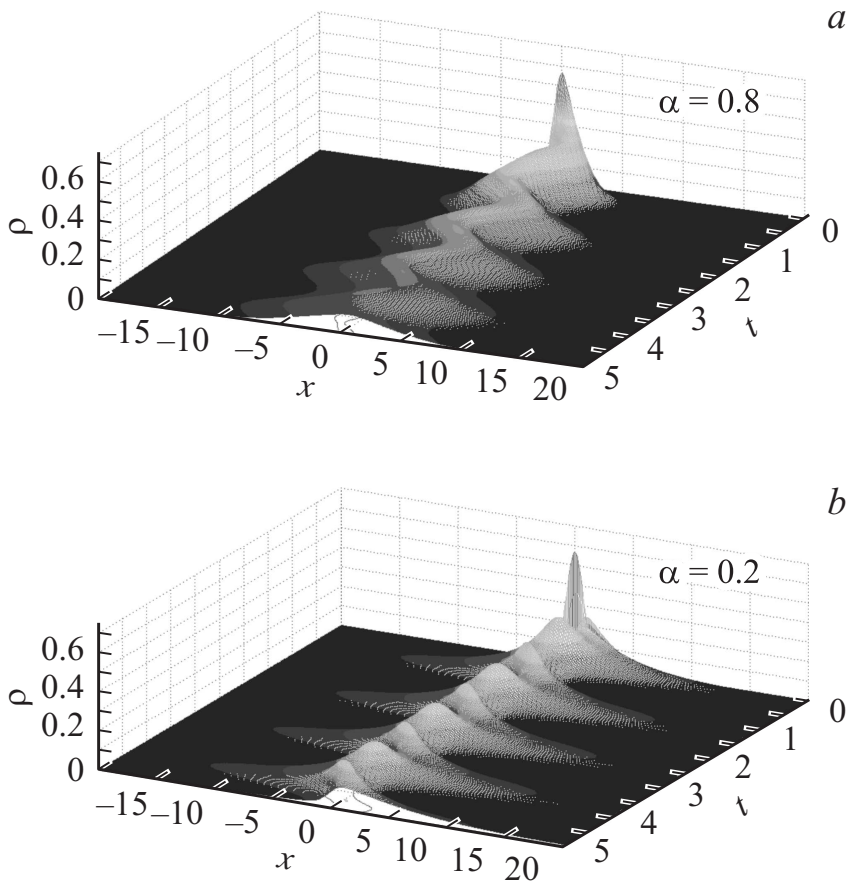

Рис. 2. Пространственно-временны́е распределения плотности Н3 при различных порядках дробной производной $(\omega=5$, $a=1)$.

приводит к локализации максимумов функции плотности около точки $x=0$. В результате в окрестности этой точки возникают осцилляции заряда по времени с удвоенной частотой по сравнению с частотой внешнего поля. На рис. 2 показано возникновение этих осцилляций.

Все описанные свойства относятся к фрактальным материалам с большими временами жизни неравновесных Н3. Если же время жизни мало, то необходимо учитывать процесс рекомбинации НЗ, что является предметом отдельного исследования.

\section{4. Заключение}

В настоящей работе показано, что порядок дробной производной по времени в дифференциальном уравнении диффузионно-дрейфовой модели связан с показателем Херста, а также с фрактальной размерностью траекторий случайного блуждания Н3 в отсутствие внешних полей. Численное моделирование показывает, что во фрактальной полупроводниковой структуре в продольном переменном электрическом поле может возникать явление удвоения частоты осцилляций заряда, обусловленное наличием памяти в кинетике процесса. По нашему мнению, это явление можно использовать для создания качественно нового умножителя частоты или электронно-оптического преобразователя для функциональной микроэлектроники. Для этих целей подходящим материалом может оказаться пористый кремний с большим временем жизни неравновесных Н3. 
Работа выполнена при поддержке гранта ОНИТ РАН № 5 „Фундаментальные проблемы физики и технологии эпитаксиальных наноструктур приборов на их основе“ и гранта президента Российской Федерации для государственной поддержки молодых российских ученых МК-3360.2015.1.

\section{Список литературы}

[1] А.И. Олемской, А.Я. Флат. УФН, 163 (12), 1 (1993).

[2] V.V. Uchaikin. Fractional Derivatives for Physicists and Engineers (Beijing, Higher Education Press, 2012).

[3] R. Herrmann. Fractional Calculus - An Introduction for Physicists (Singapore, World Scientific, 2014).

[4] V.V. Uchaikin, R.T. Sibatov. Commun. Nonlinear Sci. Numerical Simulation, 13, 715 (2008).

[5] Р.Т. Сибатов, В.В. Учайкин. УФН, 179 (10), 1079 (2009).

[6] С.Ш. Рехвиашвили, М.О. Мамчуев, М.О. Мамчуев. ФТТ, 58 (4), 763 (2016).

[7] С.Г. Самко, А.А. Килбас, О.И. Маричев. Интегралы и производные дробного порядка и некоторые их приложсения (Минск, Наука и техника, 1987).

[8] А.В. Псху. Уравнения в частных производных дробного порядка (М., Наука, 2005).

[9] Е. Федер. Фракталы (М., Мир, 1991).

[10] Р.М. Кроновер. Фракталы и хаос в динамических системах. Основы теории (М., Мир, Постмаркет, 2000).

[11] С.Ш. Рехвиашвили. Письма ЖТФ, 30 (2), 33 (2004).

[12] С.Ш. Рехвиашвили. Нелинейный мир, 5 (4), 194 (2007).

[13] А.В. Псху. Изв. РАН. Сер. мат., 73 (2), 141 (2009).

[14] В.В. Учайкин. ЖТФ, 74 (7), 123 (2004).

[15] A.A. Alikhanov. J. Comput. Phys., 280, 424 (2015).

[16] Р.Р. Нигматуллин. ТМФ, 90 (3), 354 (1992).

Редактор А.Г. Оганесян

\section{Simulation of the drift-diffusion transport of charge carriers in the semiconductor layer with the fractal structure in alternating electric field}

\section{S.Sh. Rekhviashvili, A.A. Alikhanov}

Institute of Applied Mathematics and Automation KBCC RAS, 360000 Nalchik, Russia

Abstract Based on the differential equation of fractional order a simulation of a drift-diffusion transport of charge carriers in the semiconductor layer with the fractal structure under the action of longitudinal alternating electric field has been used. The modeling shows that in layers with a fractal structure broadening and asymmetry of the spatial and temporal distributions of the charge carriers is happened. Under certain conditions, there is the effect of the frequency doubling of the charge oscillations in an external alternating electric field. 